\title{
Recent results from BABAR
}

\author{
J. William Gary ${ }^{1, *}$ \\ ${ }^{1}$ Department of Physics and Astronomy, University of California, Riverside, CA 92521, USA
}

\begin{abstract}
The BABAR Collaboration at SLAC continues to produce a wide variety of publications on interesting and unique topics despite having ceased data-collection operations more than 10 years ago. Presented here are the results of three recent studies. The first is on the direct measurement of the $B^{ \pm} \rightarrow$ $K^{ \pm} X(3872)$ branching fraction, allowing the first determination of the branching fractions of the exotic $X(3872)$ charmonium state. The second is a publication on the first observation of the $D^{0} \rightarrow K^{-} \pi^{+} e^{+} e^{-}$branching fraction. The third is on searches for lepton flavor and lepton number violating decays in $D^{0}$ meson decays, resulting in improvements over existing limits by factors that vary from 20 to almost 800 depending on the $D^{0}$ decay channel.
\end{abstract}

\section{Introduction}

The BABAR detector collected data at the SLAC PEP-II asymmetric $e^{+} e^{-}$collider from 1999 to 2008. Despite having ceased operation more than 10 years ago, the data analysis is still active, with 7-8 papers submitted to journals each of the past several years. The PEP-II collider acted as a " $B$ factory", built to study $C P$ violation in $B$ meson decays. Nonetheless, the general-purpose design of BABAR makes its data sample suitable for a wide variety of other studies, such as the ones discussed here.

Most of the BABAR data were collected at the energy of the $\Upsilon(4 S)$ resonance, which decays to a $B \bar{B}$ pair essentially $100 \%$ of the time, thus yielding a large sample of $B$ mesons. The final BABAR data sample contains around 471 million $B \bar{B}$ pairs, corresponding to an integrated luminosity of $424 \mathrm{fb}^{-1}$. Beside $B$ mesons, the PEP-II collider also produced a large number of charmed mesons, because of the strong coupling of charm quarks to the intermediate photons from the $e^{+} e^{-}$annihilation.

The main topic described here is the first measurement of the absolute $B^{ \pm} \rightarrow K^{ \pm} X(3872)$ branching fraction. Recent results on the first observation of $D^{0} \rightarrow K^{-} \pi^{+} e^{+} e^{-}$decays, and a search for rare and forbidden decays of the $D^{0}$ meson, are also described.

\section{Absolute measurement of the $B^{ \pm} \rightarrow K^{ \pm} X(3872)$ branching fraction}

This study [1] was recently submitted to Physical Review Letters.

A number of studies of exclusive 2-body $B$ meson decays of the form $B^{ \pm} \rightarrow K^{ \pm} X_{c \bar{c}}$ have been presented, where $X_{c \bar{c}}$ is a charmonium state. For example, $X_{\bar{c} \bar{c}}$ could be a $J / \psi, \chi_{c J}(1 P)$, $\eta_{c}$, or $\psi(2 S)$ meson, or an exotic state such as the $X(3872)$. A Feynman diagram is shown in Fig. 1. These studies are focused on tests of $B$ hadron dynamics and $\mathrm{CP}$ violation. Examples of such studies are given in Refs. [2, 4, 5].

\footnotetext{
*e-mail: bill.gary@ucr.edu
} 


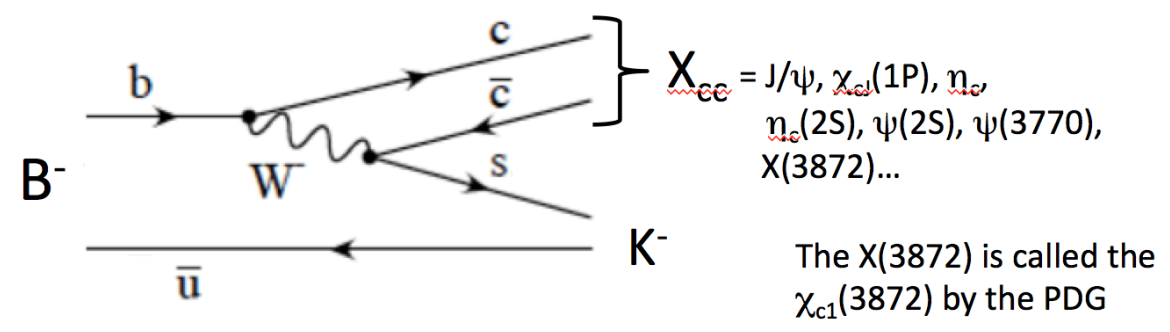

Figure 1. Feynman diagram for $B^{ \pm} \rightarrow K^{ \pm} X_{c \bar{c}}$, where $X_{c \bar{c}}$ is a charmonium state.

In these studies, the $X_{\bar{c}}$ is reconstructed in its decay to a specific final state " $f$ ". Thus these studies do not measure the absolute branching fractions $\mathcal{B}\left(B^{ \pm} \rightarrow K^{ \pm} X_{\bar{c} \bar{c}}\right)$ or $\mathcal{B}\left(X_{\bar{c} \bar{c}} \rightarrow f\right)$ but rather their product. To obtain the absolute branching fraction $\mathcal{B}\left(B^{ \pm} \rightarrow K^{ \pm} X_{c \bar{c}}\right)$ or $\mathcal{B}\left(X_{c \bar{c}} \rightarrow f\right)$, the other of the two must already be known. For well established particles like the $J / \psi, \eta_{c}$, etc., this is not a problem, because the $X_{c \bar{c}} \rightarrow f$ branching fractions of these particles are well known, and the focus of these studies is on the $B$ hadron branching fraction $\mathcal{B}\left(B^{ \pm} \rightarrow K^{ \pm} X_{c \bar{c}}\right)$. However, for the exotic charmonium state $X(3872)$, the absolute branching fractions $\mathcal{B}(X(3872) \rightarrow f)$ are not known. The $X(3872)$ has only been observed in the decay $B^{ \pm} \rightarrow K^{ \pm} X(3872)$ followed by the decay to some specific reconstructed final state $X(3872) \rightarrow f$, and we therefore only know the product $\mathcal{B}\left(B^{ \pm} \rightarrow K^{ \pm} X(3872)\right) \mathcal{B}(X(3872) \rightarrow$ $f)$. However, knowledge of the absolute values of $\mathcal{B}(X(3872) \rightarrow f$ ) is of significant importance because it would greatly aid in understanding the nature of the $X(3872)$, as discussed in the following paragraph. The new BABAR analysis aims to measure the absolute $B^{ \pm} \rightarrow K^{ \pm} X(3872)$ branching fraction and thus, in conjunction with the already known product $\mathcal{B}\left(B^{ \pm} \rightarrow K^{ \pm} X(3872)\right) \mathcal{B}(X(3872) \rightarrow f)$, allow the absolute $X(3872) \rightarrow f$ branching fractions to be determined.

A multitude of exotic charmonium states have been discovered over the past two decades. Their masses and/or decay properties do not correspond to those expected for yet-undiscovered non-exotic states and thus they do not fit into the framework of normal $c \bar{c}$ mesons. These exotic charmonium states have been dubbed the " $X$ ", " $Y$ ", and " $Z$ " particles. The $Y$ particles are those with $J^{P C}=1^{-}$, the $Z$ particles are those with isospin 1 , and the $X$ particles are all the rest [3]. The $X(3872)$ was the first $X Y Z$ particle discovered [4] and is the most studied. There are two main interpretations of the $X(3872)$ : that it is a $D^{0} \bar{D}^{* 0}$ molecule, namely two color singlet systems loosely bound together [6], or that it is a point-like tetraquark, namely two tightly bound color triplet systems [7]. Other interpretations include a $\chi_{c 1}(2 P)-D D^{*}$ hybrid or a $c \bar{c}$-gluon hybrid. (See Refs. [3, 8] for reviews.) For the decay $X(3872) \rightarrow J / \psi \pi^{+} \pi^{-}$, which is the $X(3872)$ discovery channel [4], the simple tetraquark model predicts a large branching fraction, $\mathcal{B}\left(X(3872) \rightarrow J / 4 \pi^{+} \pi^{-}\right) \gtrsim 50 \%$ [6], while the $D^{0} \bar{D}^{* 0}$ molecular model predicts a small one, $\mathcal{B}\left(X(3872) \rightarrow J / \psi \pi^{+} \pi^{-}\right) \approx 10 \%$ [9]. Thus, knowledge of the absolute branching fractions of the $X(3872)$ allows the different interpretations to be distinguished and can greatly aid in understanding the nature of the $X(3872)$.

The $X(3872)$ has a narrow width, less than $1.2 \mathrm{MeV}$ [10], making it a good candidate for a search in a missing-mass analysis. This is the strategy of the new BABAR study, implemented as follows. It employs the well-established and very powerful hadronic tag method [11], unique to $e^{+} e^{-}$experiments and illustrated in Fig. 2. Events are selected in which one of the $B$ mesons in the $e^{+} e^{-} \rightarrow \Upsilon(4 S) \rightarrow B \bar{B}$ events can be fully reconstructed in one of its hadronic decay modes (it therefore has no undetected momentum from a neutrino). This fully 


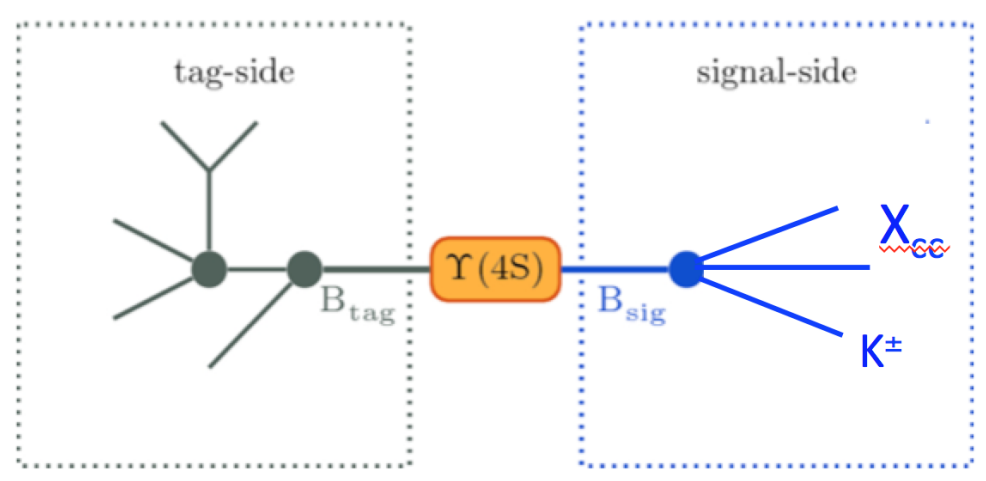

Figure adapted from Keck, T., Abudinén et al. Comput Softw Big Sci (2019) 3

Figure 2. Illustration of the hadronic tag method to determine $\mathcal{B}\left(B^{ \pm} \rightarrow K^{ \pm} X_{\bar{c}}\right)$.

reconstructed $B$ meson is denoted $B_{t a g}$. One then retains those events with an identified $K^{ \pm}$in the other $B$ meson. This other $B$ meson is denoted $B_{\text {sig }}$, i.e., it is the signal $B$ meson. Since the energies and momenta of the initial $e^{+}$and $e^{-}$are completely known, as are those of $B_{\text {tag }}$, so are the energy and momentum of the $B_{\text {sig }}$ meson, and one can thus boost to the center-of-mass (c.m.) of $B_{s i g}$ and plot the momentum $p_{K}^{\text {c.m. }}$ of the $K^{ \pm}$in this frame. The momentum $p_{K}^{\text {c.m. }}$ in the two-body $B^{ \pm} \rightarrow K^{ \pm} X_{c \bar{c}}$ events is related to the missing mass $m_{X}$ recoiling against the $K^{ \pm}$ through the relation $m_{X}=\sqrt{m_{B}^{2}+m_{K}^{2}-2 E_{K}^{\text {c.m. }} m_{B}}$, with $E_{K}^{\text {c.m. }}$ the c.m. energy of the $K^{ \pm}$. We choose to examine $p_{K}^{\text {c.m. }}$ rather than $m_{X}$ because the experimental resolution is better. If a peak is observed at the $p_{K}^{\text {c.m. }}$ value corresponding to the $X(3872)$ mass, one can thereby determine the absolute $B^{ \pm} \rightarrow K^{ \pm} X(3872)$ branching fraction directly, with no knowledge required of the $X(3872) \rightarrow f$ branching fractions, since the $X(3872)$ is not reconstructed but rather detected only through its recoil. Then the $X(3872) \rightarrow f$ branching fractions can be determined as explained above, from the already known product $\mathcal{B}\left(B^{ \pm} \rightarrow K^{ \pm} X(3872)\right) \mathcal{B}(X(3872) \rightarrow f)$.

In fact, this missing mass method to search for the $X(3872)$ has already been used twice previously, but without success. The technique was pioneered by BABAR in 2006 [12] using a data sample of $211 \mathrm{fb}^{-1}$. No signal was seen and the limit $\mathcal{B}\left(B^{ \pm} \rightarrow K^{ \pm} X(3872)\right)<3.2 \times 10^{-4}$ was obtained. The Belle experiment repeated the study in 2018 [13], similarly not observing a signal, and improving the limit to $\mathcal{B}\left(B^{ \pm} \rightarrow K^{ \pm} X(3872)\right)<2.6 \times 10^{-4}$.

The new BABAR study makes use of the final BABAR data set, corresponding to $424 \mathrm{fb}^{-1}$, thereby doubling the data sample compared to the 2006 analysis. More importantly, the $X(3872)$ signal efficiency has been improved by a factor of 3 compared to 2006, primarily because, in the new analysis, all $B_{t a g}$ candidates in an event are accepted, in contrast to the previous BABAR and Belle studies, for which only the best $B_{t a g}$ candidate (one per event) was accepted. In the new study, the mean number of $B_{t a g}$ candidates, for events that have at least one such candidate, is 1.9 . Thus by accepting all $B_{\text {tag }}$ candidates, and not merely the best one, the $X(3872)$ signal efficiency is improved by about a factor of 2 . In the previous analysis, the signal $B$ meson (i.e., the reconstructed $B$ meson containing the $K^{ \pm}$) was often the best $B_{\text {tag }}$ candidate as well, and was retained exclusively as such, thus removing it from the signal candidate pool. The remainder of the factor of 3 improvement in the $X(3872)$ signal efficiency relative to the 2006 study is achieved through improvements in the hadronic tag and background-subtraction procedures. Thus, the new analysis is optimized for the $X(3872)$ signal efficiency rather than the $B_{\text {tag }}$ purity. 


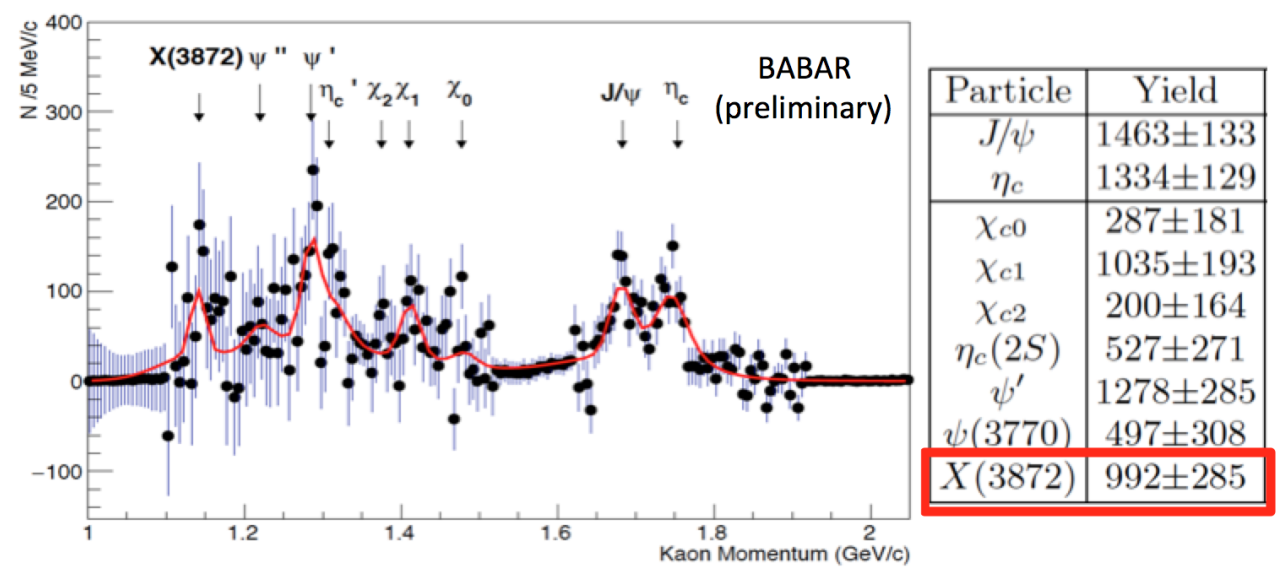

Figure 3. The background-subtracted distribution of the $K^{ \pm}$momentum in $B_{\text {sig }}$ signal $B$ mesons, plotted in the $B_{s i g}$ center-of-mass. The curve shows the result of a maximum likelihood fit to the data, accounting for the resonances shown in the table to the right. The table shows the fitted signal yield of each resonance, with its statistical uncertainty.

The results for the $p_{K}^{\text {c.m. }}$ spectrum in the selected events are shown in Fig. 3. A clear peak is observed for the $X(3872)$, corresponding to $p_{K}^{\text {c.m. }} \approx 1.13 \mathrm{GeV} / c^{2}$. We thus obtain the first signal for the $X(3872)$ using a missing-mass technique. A maximum likelihood fit of the background-subtracted data is performed to determine the yields of the observed resonances. We find $992 \pm 285 X(3872)$ candidates, as indicated in the table on the right side of Fig. 3. By repeating the fit with the $X(3872)$ signal yield fixed to zero, the significance of the $X(3872)$ signal, after accounting for the effects of systematic uncertainties, is found to be 3.0 standard deviations.

From the observed yield, we determine the absolute $B^{ \pm} \rightarrow K^{ \pm} X_{c \bar{c}}$ branching fraction to be $\mathcal{B}\left(B^{ \pm} \rightarrow K^{ \pm} X(3872)\right)=(2.1 \pm 0.7) \times 10^{-4}$. In conjunction with the result for the product $\mathcal{B}\left(B^{ \pm} \rightarrow K^{ \pm} X(3872)\right) \mathcal{B}\left(X(3872) \rightarrow J / \psi \pi^{+} \pi^{-}\right)=(8.6 \pm 0.8) \times 10^{-6}$ [10], we thus obtain $\mathcal{B}\left(X(3872) \rightarrow J / \psi \pi^{+} \pi^{-}\right)=(4.1 \pm 1.3) \%$ (preliminary). This rules out the simple tetraquark model, which predicts $\mathcal{B}\left(X(3872) \rightarrow J / \psi \pi^{+} \pi^{-}\right) \gtrsim 50 \%$ as mentioned above. Molecular models, with $\mathcal{B}\left(X(3872) \rightarrow J / \psi \pi^{+} \pi^{-}\right) \approx 10 \%$ or less, are much more consistent with our measurement. We should note, however, that the pure molecular model has difficulty describing other results, such as the branching fractions in $X(3872)$ radiative decays.

\section{First observation of the rare decay $D^{0} \rightarrow K^{-} \pi^{+} e^{+} e^{-}$}

Searches for rare decay modes of particles are sensitive to new physics, which can modify the decay rates and angular distributions (thus the detection efficiencies). Since the standard model (SM) decay rate is small or negligible for rare processes, the new physics contributions are not necessarily overwhelmed and might be visible. The decay $D^{0} \rightarrow K^{-} \pi^{+} e^{+} e^{-}$ is rare in the SM. It cannot occur at tree level. Short distance contributions occur through box and penguin diagrams, with SM branching fractions too small $\left(O\left(10^{-9}\right)\right)$ [14] to be currently detectable. Long distance contributions from photon pole or vector meson dominance processes can increase the SM branching fraction up to $O\left(10^{-6}\right)$ [14]. New physics such as supersymmetry might substantially increase the rate $[14,15]$. The current limit on the branching fraction is $\mathcal{B}\left(D^{0} \rightarrow K^{-} \pi^{+} e^{+} e^{-}\right)<4.1 \times 10^{-5}[16]$. 

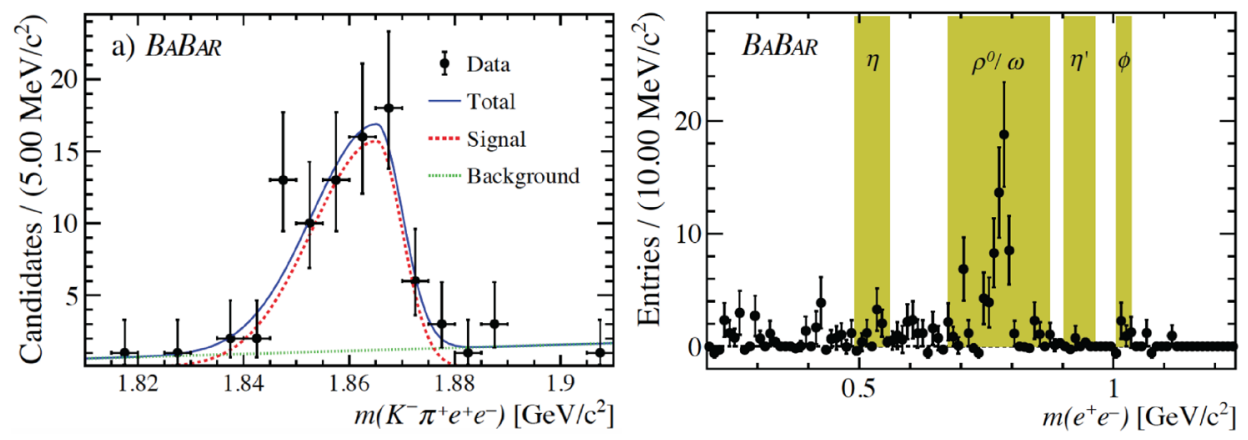

Figure 4. (Left) Reconstructed $D^{0} \rightarrow K^{-} \pi^{+} e^{+} e^{-}$invariant mass peak. (Right) Invariant mass distribution $m\left(e^{+} e^{-}\right)$of the final state $e^{+} e^{-}$pair in $D^{0} \rightarrow K^{-} \pi^{+} e^{+} e^{-}$. The shaded regions are excluded in the search for $D^{0} \rightarrow K^{-} \pi^{+} e^{+} e^{-}$from short-distance interactions.

We perform a search [17] for $D^{0} \rightarrow K^{-} \pi^{+} e^{+} e^{-}$based on a data sample of $468 \mathrm{fb}^{-1}$ and find $68 \pm 9$ signal events. The signal peak is shown in Fig. 4 (left). The background is seen to be very small. The significance of the signal is $>9$ standard deviations. This is the first observation of this decay mode. We find $\mathcal{B}\left(D^{0} \rightarrow K^{-} \pi^{+} e^{+} e^{-}\right)=(4.0 \pm 0.5) \times 10^{-6}$, consistent with the expectation for long-distance SM interactions.

To search away from regions dominated by long distance interactions, where the $e^{+} e^{-}$ in the final state arises from vector meson decay $\left(\eta, \rho^{0}, \eta^{\prime}\right.$, and $\left.\phi\right)$, we repeat the search excluding the invariant mass $m\left(e^{+} e^{-}\right)$regions indicated by the shaded bands in Fig. 4 (right). This allows us to probe regions where short-distance interactions might dominate. We do not observe a significant signal and set the limit $\mathcal{B}\left(D^{0} \rightarrow K^{-} \pi^{+} e^{+} e^{-}\right)_{\text {non-resonant }}<3.1 \times 10^{-6}$.

\section{Search for rare or forbidden decays of the $D^{0}$ meson}

\begin{tabular}{|c|c|c|c|c|c|}
\hline $\begin{array}{l}\text { Decay mode } \\
D^{0} \rightarrow\end{array}$ & $\begin{array}{c}N_{\text {sig }} \\
\text { (candidates) }\end{array}$ & $\begin{array}{c}\epsilon_{\mathrm{sig}} \\
(\%)\end{array}$ & $\begin{array}{c}\mathcal{B} \\
\left(\times 10^{-7}\right)\end{array}$ & $\begin{array}{r}\mathcal{B}_{90 \%}^{\text {U.L. }} \\
\left(\times 10^{-7}\right)\end{array}$ & $\begin{array}{r}\mathcal{B}_{90 \%}^{\text {U.L. }}[\mathrm{PDG}] \\
\left(\times 10^{-7}\right)\end{array}$ \\
\hline$\pi^{-} \pi^{-} e^{+} e^{+}$ & $0.22 \pm 3.15 \pm 0.54$ & 4.38 & $0.27 \pm 3.90 \pm 0.67$ & 9.1 & 1120 \\
\hline$\pi^{-} \pi^{-} \mu^{+} \mu^{+}$ & $6.69 \pm 4.88 \pm 0.80$ & 4.91 & $7.40 \pm 5.40 \pm 0.91$ & 15.2 & 290 \\
\hline$\pi^{-} \pi^{-} e^{+} \mu^{+}$ & $12.42 \pm 5.30 \pm 1.45$ & 4.38 & $15.41 \pm 6.59 \pm 1.85$ & 30.6 & 790 \\
\hline$\pi^{-} \pi^{+} e^{ \pm} \mu^{\mp}$ & $1.37 \pm 6.15 \pm 1.28$ & 4.79 & $1.55 \pm 6.97 \pm 1.45$ & 17.1 & 150 \\
\hline$K^{-} \pi^{-} e^{+} e^{+}$ & $-0.23 \pm 0.97 \pm 1.28$ & 3.19 & $-0.38 \pm 1.60 \pm 2.11$ & 5.0 & $28^{\dagger}$ \\
\hline$K^{-} \pi^{-} \mu^{+} \mu^{+}$ & $-0.03 \pm 2.10 \pm 0.40$ & 3.30 & $-0.05 \pm 3.34 \pm 0.64$ & 5.3 & 3900 \\
\hline$K^{-} \pi^{-} e^{+} \mu^{+}$ & $3.87 \pm 3.96 \pm 2.36$ & 3.48 & $5.84 \pm 5.97 \pm 3.56$ & 21.0 & 2180 \\
\hline$K^{-} \pi^{+} e^{ \pm} \mu^{\mp}$ & $2.52 \pm 4.60 \pm 1.35$ & 3.65 & $3.62 \pm 6.61 \pm 1.95$ & 19.0 & 5530 \\
\hline$K^{-} K^{-} e^{+} e^{+}$ & $0.30 \pm 1.08 \pm 0.41$ & 3.25 & $0.43 \pm 1.54 \pm 0.58$ & 3.4 & 1520 \\
\hline$K^{-} K^{-} \mu^{+} \mu^{+}$ & $-1.09 \pm 1.29 \pm 0.42$ & 6.21 & $-0.81 \pm 0.96 \pm 0.32$ & 1.0 & 940 \\
\hline$K^{-} K^{-} e^{+} \mu^{+}$ & $1.93 \pm 1.92 \pm 0.83$ & 4.63 & $1.93 \pm 1.93 \pm 0.84$ & 5.8 & 570 \\
\hline$K^{-} K^{+} e^{ \pm} \mu^{\mp}$ & $4.09 \pm 3.00 \pm 1.59$ & 4.83 & $3.93 \pm 2.89 \pm 1.45$ & 10.0 & 1800 \\
\hline
\end{tabular}

Figure 5. Results for the branching fractions of lepton flavor violating and lepton number violating decays of the $D^{0}$ meson. 
Lepton flavor violation (LFV) and lepton number violation (LNV) are essentially forbidden in the standard model but arise in many models of new physics. The BABAR data sample, with its large number of charmed mesons and well reconstructed events, is ideally suited for a search for LFV and LNV in $D^{0}$ meson decays. Figure 5 shows the results obtained in our recent search [18] for such decays. No significant signal for LFV or LNV is found. The table in this figure presents the decay mode, the number of observed candidates, the detection efficiency, the branching fraction, the $90 \%$ confidence level upper limit on the branching fraction, and the current such limit from the Particle Data Group [10]. Where uncertainties are given, the first is statistical and the second systematic. The BABAR results improve on the current limits by very large factors: from around 20 to almost 800 depending on the channel.

\section{Summary}

The BABAR Collaboration continues to produce a wide variety of publications on interesting and unique topics despite having ceased data-collection operations more than 10 years ago. Presented here are the results of a study that provides the first direct measurement of the $B^{ \pm} \rightarrow$ $K^{ \pm} X(3872)$ branching fraction, allowing the first determination of the branching fractions of the exotic $X(3872)$ charmonium state. Also presented are the results of a publication on the first observation of the $D^{0} \rightarrow K^{-} \pi^{+} e^{+} e^{-}$branching fraction, and the results of a search for lepton flavor and lepton number violating decays in $D^{0}$ meson decays that improves existing limits by factors from 20 to almost 800 , depending on the $D^{0}$ decay channel.

\section{References}

[1] BABAR Collaboration, arXiv:1911.11740.

[2] Belle Collaboration, Phys. Rev. Lett 89, 102001 (2002); Phys. Rev. D 67, 032003 (2003); Phys. Rev. Lett. 90, 071801 (2003).

[3] S. Olsen et al., Rev. Mod. Phys. 90, 015003 (2018).

[4] Belle Collaboration, Phys. Rev. Lett. 91, 262001 (2003).

[5] BABAR Collaboration, Phys. Rev. D 65, 032001 (2002); Phys. Rev. Lett. 94, 141901 (2005); Phys. Rev. D 93, 071103 (2004).

[6] N. Toernqvist, Phys. Lett. B 590, 209 (2004).

[7] L. Maiani et al., Phys. Rev. D 71, 014028 (2005); Phys. Lett. B 778, 247 (2018).

[8] H. X. Chen et al., Phys. Rep. 639, 1 (2016).

[9] E. Braaten et al., Phys. Rev. D 72, 054002 (2005).

[10] Particle Data Group, Phys. Rev. D 98, 030001 (2018).

[11] BABAR Collaboration, Phys. Rev. Lett. 92, 071802 (2004); Phys. Rev. D 88, 072012 (2013).

[12] BABAR Collaboration, Phys. Rev. Lett. 96, 052002 (2006).

[13] Belle Collaboration, Phys. Rev. D 97, 012005 (2018).

[14] S. Fajfer, S. Prelovsek, and P. Singer, Phys. Rev. D. 64, 114009 (2001).

[15] G. Burdman et al., Phys. Rev. D 66, 014009 (2002).

[16] BESIII Collaboration, Phys. Rev. D 97, 072015 (2018).

[17] BABAR Collaboration, Phys. Rev. Lett. 122, 081802 (2019).

[18] BABAR Collaboration, arXiv:1905.00608. 